\title{
Obtaining Information about Operation of Centrifugal Compressor from Pressure by Combining EEMD and IMFE
}

\author{
Yan Liu *, Kai Ma, Hao He and Kuan Gao \\ School of Mechanical Engineering, Northwestern Polytechnical University, Xi'an 710072, China; \\ makai@mail.nwpu.edu.cn (K.M.); haohe@mail.nwpu.edu.cn (H.H.); 15991737832@mail.nwpu.edu.cn (K.G.) \\ * Correspondence: liuyan@nwpu.edu.cn; Tel.: +86-298-859-4983
}

Received: 18 March 2020; Accepted: 7 April 2020; Published: 9 April 2020

check for updates

\begin{abstract}
Based on entropy characteristics, some complex nonlinear dynamics of the dynamic pressure at the outlet of a centrifugal compressor are analyzed, as the centrifugal compressor operates in a stable and unstable state. First, the $800-\mathrm{kW}$ centrifugal compressor is tested to gather the time sequence of dynamic pressure at the outlet by controlling the opening of the anti-surge valve at the outlet, and both the stable and unstable states are tested. Then, multi-scale fuzzy entropy and an improved method are introduced to analyze the gathered time sequence of dynamic pressure. Furthermore, the decomposed signals of dynamic pressure are obtained using ensemble empirical mode decomposition (EEMD), and are decomposed into six intrinsic mode functions and one residual signal, and the intrinsic mode functions with large correlation coefficients in the frequency domain are used to calculate the improved multi-scale fuzzy entropy (IMFE). Finally, the statistical reliability of the method is studied by modifying the original data. After analysis of the relationships between the dynamic pressure and entropy characteristics, some important intrinsic dynamics are captured. The entropy becomes the largest in the stable state, but decreases rapidly with the deepening of the unstable state, and it becomes the smallest in the surge. Compared with multi-scale fuzzy entropy, the curve of the improved method is smoother and could show the change of entropy exactly under different scale factors. For the decomposed signals, the unstable state is captured clearly for higher order intrinsic mode functions and residual signals, while the unstable state is not apparent for lower order intrinsic mode functions. In conclusion, it can be observed that the proposed method can be used to accurately identify the unstable states of a centrifugal compressor in real-time fault diagnosis.
\end{abstract}

Keywords: centrifugal compressor; surge; nonlinear dynamics; multi-scale fuzzy entropy; ensemble empirical mode decomposition

\section{Introduction}

In recent years, centrifugal compressors have been used widely in industry. As an intrinsic characteristic of the centrifugal compressor, surge can cause flow-induced vibration and lower aerodynamic performance. Therefore, surge may increase the energy consumption of a centrifugal compressor and lead to fatigue of the structure or instability of aeroelasticity as the surge becomes violent [1,2].

Researchers have completed a lot of studies on surge, as energy-saving is required and centrifugal compressors are used in variable working conditions. Roughly speaking, there are two ways to study surge, namely, mechanism studies and identification from the data. In mechanism studies, it is believed that an external factor and two internal factors may induce surge. The external factor is high-pressure gas stored in the pipes, and the internal factors are rotation stall in the passage and separation of 
flow around the blades. Because the dynamic pressure at the outlet of a centrifugal compressor can indicate the state of the compressor accurately and immediately, it provides a way to study surge. In particular, the dynamic pressure becomes complex and seriously disordered as the centrifugal compressor undergoes transition from the stable state to unstable states, and routine methods, such as spectral analysis and time-domain analysis, fail to capture and describe this complex behavior exactly.

With the development of nonlinear dynamics, various nonlinear analysis methods have been widely applied in the surge prediction of centrifugal compressors, such as wavelet analysis, Lyapunov exponent, and fractal dimension [3-6]. The studies show that the flow from the inlet, impeller, and to the outlet behaves chaotically or disorderly as the working state of a centrifugal compressor is unstable. That is, the time sequence of the flow pressure becomes singular in the unstable state, and therefore some intrinsic properties should exist in the time sequence. Fortunately, entropy can be used to describe and measure such a singular phenomenon. Indeed, information entropy has been introduced to characterize dynamical systems between purely random, chaotic, and regular evolution [7-9]. In 2008, Chen and his group proposed the concept of fuzzy entropy (FE) and used the method to extract the characteristic information of a surface electromyogram signal $[10,11]$. Because of the many advantages of $\mathrm{FE}$, it is widely used in economic management, medical diagnosis, weather forecasting, biology, ecology, and other fields [12,13]. Since then, some other methods are developed on the basis of entropy. Among them, multi-scale fuzzy entropy (MFE), as one method of the fuzzy entropy family, has advantages in analyzing a dynamical system by placing the sample data on different scales [14-17]. Similarly, ensemble empirical mode decomposition (EEMD) is also powerful in dealing with non-stationary and nonlinear data. Based on the scale feature of the signal itself, EEMD could decompose a signal into several intrinsic mode functions, in order to achieve better analysis performance [18]. In particular, it is suitable for analyzing nonlinear and non-stationary signal sequences because of its high signal-to-noise ratio. Hence, a method combining EEMD and MFE can be considered as a strategy to capture the intrinsic properties of dynamic pressure with different orders, especially for the dynamic pressure as a centrifugal compressor operates in an unstable state [19].

In this study, some fundamental theories related to MFE, improved multi-scale fuzzy entropy (IMFE), and EEMD, are used and developed to describe the dynamic pressure of flow at the outlet of a centrifugal compressor. Then, a method combining IMFE with EEMD is proposed to measure the complexity of the intrinsic mode function of dynamic pressure under different scale factors and the probability of new information with the changes of dimension. Finally, some conclusions are obtained, and the feasibility of the method is verified to identify surge.

\section{Fundamental Theories}

\subsection{Multi-Scale Fuzzy Entropy and Improved Multi-Scale Fuzzy Entropy}

Entropy is a concept that originated from the field of thermodynamics, and Shannon first applied entropy to the field of information theory and proposed the concept of information entropy to measure the uncertainty of an event [20]. Since then, the concept of entropy has gradually been generalized. According to functions and applications, entropy can be divided into approximate entropy, sample entropy, fuzzy entropy, and so on. Among these methods, FE can describe clearly the edge of adjacent classes of sample data, which is a weakness of information entropy, approximate entropy, and sample entropy. In particular, FE could accurately describe the complexity of a system, because of its strong consistency and reduced dependence on the length of data. With the advantages of small deviation, good continuity, free parameter selection, and strong anti-interference ability, FE is suitable for studying dynamic pressure at the outlet of a centrifugal compressor [8]. Multi-scale fuzzy entropy, as a special kind of fuzzy entropy, gives a measurement of the complexity of a sequence under different scale factors [15]. Hence, both MFE and IMFE are used in the paper to investigate the complex nonlinear dynamic characteristics of dynamic pressure at the outlet of a centrifugal compressor, and identify the unstable state of the system. 
For time sequence $x$, FE, MFE and IMFE can be calculated as follows [21-23],

1 . For an $N$-length time sequence $x(i), i=(1,2, \ldots, N)$, construct $m$-dimension vector for $x(i)$, with $m$ being the embedded dimension,

$$
X_{j}^{m}=\{x(j), x(j+1), \ldots, x(j+m-1)\}-x_{0}(j), 1 \leq j \leq N-m+1
$$

where

$$
x_{0}(j)=\frac{1}{m} \sum_{k=0}^{m-1} x(j+k)
$$

2. Define the distance $d_{j l}^{m}$ between $X_{j}^{m}$ and $X_{l}^{m}$ as the maximum value of the difference between the two elements,

$$
\begin{aligned}
& d_{j l}^{m}=d\left[X_{j}^{m}, X_{l}^{m}\right]=\max _{k \in(0, m-1)}\left\{\left|x(j+k)-x_{0}(j)\right|-\left|x(l+k)-x_{0}(l)\right|\right\} \\
& 1 \leq j \leq N-m+1,1 \leq l \leq N-m+1, j \neq l
\end{aligned}
$$

3. Calculate the similarity of $d_{j l}^{m}$ by selecting the exponential function $\exp \left(-\ln (2) \cdot\left(d_{j l}^{m} / r\right)^{n}\right)$ as a fuzzy function, where $n$ and $r$ are the gradient and width of the boundary, respectively. The similarity of $\Phi^{m}$ can be defined as follows,

$$
\Phi^{m}(n, r)=\frac{1}{N-m+1} \sum_{j=1}^{N-m+1} \frac{1}{N-m} \sum_{l=1, l \neq j}^{N-m+1} \exp \left(-\ln (2) \cdot\left(d_{j l}^{m} / r\right)^{n}\right)
$$

4. Repeat Equations (1)-(4), for obtaining $m+1$ dimensional similarity, and $\Phi^{m+1}$ can be described.

5. Fuzzy entropy is defined as the negative natural logarithm of the ratio of $\Phi^{m}$ and $\Phi^{m+1}$, FE for a time sequence $x(i)$ is shown in Equation (5),

$$
\mathrm{FE}(x(i), m, n, r)=-\ln \left(\frac{\Phi^{m+1}(n, r)}{\Phi^{m}(n, r)}\right)
$$

6. In order to calculate MFE, the coarse-grained data are obtained following Equation (6),

$$
y_{o}^{\tau}=\frac{1}{\tau} \sum_{i=(o-1) \tau+1}^{o \tau} x(i), 1 \leq o \leq \frac{N}{\tau}
$$

where $\tau$ is the scale factor. The coarse-grained data of MFE with two and three scale factors are shown in Figure 1.

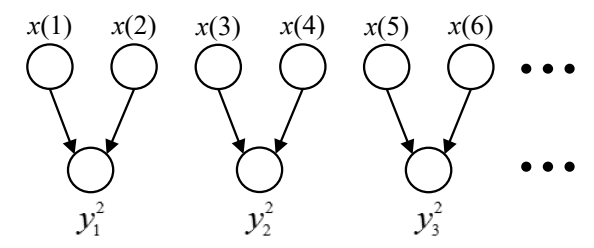

(a) Two-scale factors

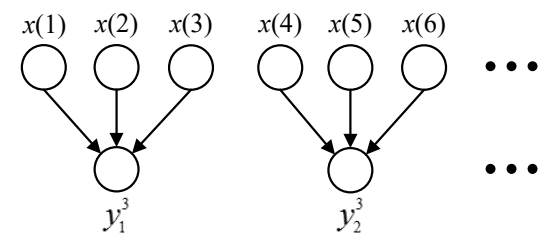

(b) Three-scale factors

Figure 1. Coarse-grained data of multi-scale fuzzy entropy (MFE) with scale factors. 
Further, MFE for a time sequence $x(i)$ is expressed as Equation (7),

$$
\operatorname{MFE}(x(i), m, n, r, \tau)=\operatorname{FE}\left(y_{o}^{\tau}, m, n, r\right)
$$

It should be noted that there are some shortcomings in MFE. First, MFE does not exactly correspond to the original time sequence in its complexity, and some characteristics in the original time sequence may be lost. Another shortcoming is the variability of the entropy results for high scale factors, because the time sequence becomes shorter as the scale factor increases. These may lead to an unstable measure of entropy.

To reduce these defects, Hamed proposed a new method named IMFE to obtain coarse-grained data, instead of the mean value method in MFE $[23,24]$. Coarse graining of improved multi-scale fuzzy entropy is expressed as Equation (7). The coarse-grained data of IMFE with two- and three-scale factors are shown in Figure 2. The differences between the data points and their corresponding averages become even more striking for the coarse-grained data of IMFE than those of MFE.

$$
z_{p}^{\tau}=\frac{1}{\tau} \sum_{i=p}^{p+\tau-1} x(i), 1 \leq p \leq N-\tau+1
$$

According to Equation (8), the original time sequence is coarse-grained for IMFE, and IMFE for a time sequence $x(i)$ can be obtained following Equation (9),

$$
\operatorname{IMFE}(x(i), m, n, r, \tau)=\mathrm{FE}\left(z_{p}^{\tau}, m, n, r\right)
$$

Subsequently, IMFE could be calculated, which can measure or describe the self-similarity and complexity of a time sequence under different scale factors. If the entropy of a sequence is larger than that of another sequence on most scales, the former is considered to be more complex than the latter. Moreover, if the entropy of a time sequence decreases monotonically with the increase of scale factor, it indicates that the structure of the time sequence is simple, and the main information is included in the entropy of the minimum scale factor.

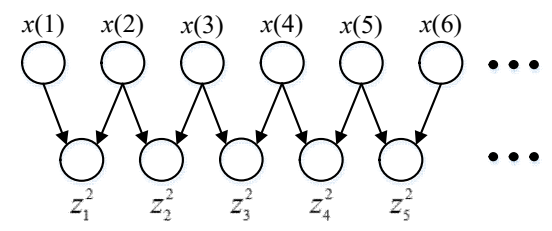

(a) Two scale factors

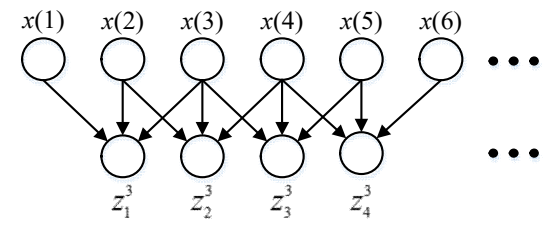

(b) Three scale factors

Figure 2. Coarse-grained data of improved multi-scale fuzzy entropy (IMFE) with scale factors.

From the definition of fuzzy entropy, it is clear that the calculation of fuzzy entropy is related to embedding dimension $m$, gradient $n$, and width $r$ of the similar boundary tolerance, and the length $N$ of data. For large values of embedding dimension $m$, a large number of data points $\left(N=10^{m}-30^{m}\right)$ is needed to obtain an accurate result. Therefore, the embedding dimension $m$ is generally set at two initially, and more detailed information can be described with an increase of $m$, as the sequence is reconstructed. For the width of the boundary, it is vital to choose a suitable $r$. For large $r$, a lot of statistical data will be lost. For small $r$, the estimated statistical characteristics are unsatisfactory and the anti-noise capability becomes weak. Therefore, the range of $r$ is generally selected to be from $0.1 S D$ 
to $0.25 \mathrm{SD}$, where $\mathrm{SD}$ is the standard deviation of the original data. After considering the characteristics of the dynamic pressure at outlet and the sample frequency, in this paper, the main parameters of IMFE are set as $r=0.15 S \mathrm{D}, n=2$, and $N=2048$, respectively. Furthermore, in order to ensure that the value of fuzzy entropy is not affected by the length of coarse-grained data, the maximum of the scale factor $\tau$ is generally ten, since the length of the coarse-grained data is $N / \tau$ for data of length $N$.

\subsection{Ensemble Empirical Mode Decomposition}

Huang proposed a time-frequency analysis method named the Hilbert-Huang Transform (HHT), which is a method combining empirical mode decomposition (EMD) and the Hilbert transform [18]. EMD is a powerful tool used to analyze a nonlinear and non-flat signal, which can decompose the signal into a series of single component signals named intrinsic mode functions (IMFs). Every IMF represents an approximate simple harmonic oscillation function with a different frequency, and these IMF components contain all the frequency components of the original signal. However, there is modal aliasing in EMD, which will lead to reduced accuracy of IMF and the loss of physical significance of IMF. Following this, $\mathrm{Wu}$ and his colleagues proposed ensemble empirical mode decomposition (EEMD), on the basis of EMD [14]. Hence, the combination of EEMD and IMFE will be used to analyze the dynamic pressure at outlet of a centrifugal compressor.

The main steps of EEMD can be summarized as follows [18],

1. Add white noise $w(i)$ to the original signal $x(i)$,

$$
x_{q}(i)=x(i)+w_{q}(i), q=1, \ldots, M
$$

where $x_{q}(i)$ is the randomly shuffled signal at the $q$ th time, and $M$ is the maximum time.

2. Decompose $x_{q}(i)$ into $k$ IMF components and a residual $R_{q}(i)$,

$$
x_{q}(i)=\sum_{j=1}^{k} c_{q j}(i)+R_{q}(i)
$$

where $c_{q j}(i)$ is the $j$ th IMF component of $x_{q}(i)$, it is calculated by using EMD method

3. In order to eliminate effects of multiple adding white noise on IMF components, the average values of the IMF components are calculated as follows. Then, the new IMF components $C_{j}(i)$ and the remainder $R(i)$ are obtained,

$$
\begin{aligned}
& C_{j}(i)=\frac{1}{M} \sum_{q=1}^{M} c_{q j}(i) \\
& R(i)=\frac{1}{M} \sum_{q=1}^{M} R_{q}(i)
\end{aligned}
$$

In Equation (12), $C_{j}(i)$ is the $j$ th IMF component of $x(i)$. If $M$ is large enough, the sum of IMFs corresponding to white noise tends to zero. So, the original signal $x(i)$ is decomposed as follows,

$$
x(i)=\sum_{j=1}^{k} C_{j}(i)+R(i)
$$

In this paper, the amplitude of white noise is set as 0.1 PSI (Pounds per square inch, a unit of pressure) and $M=400$.

\section{Data Acquisition of Dynamic Pressure}

The diagram of the data acquisition system is shown in Figure 3. In the system, the centrifugal compressor is driven by an $800 \mathrm{~kW}$ DC motor. The number of diffuser blades is 24 , the number of 
impeller blades is 16, and the Mach number of the airflow in the pipeline is 0.6. Firstly, the outside air enters the pipeline through the intake filter, inner nozzle and chamber. After this, the filtered air is compressed by the centrifugal compressor. Finally, the compressed air is expelled into atmosphere through the exhaust muffler and outlet pipe by controlling the opening-degree of the two electric anti-surge valves with diameters of $250 \mathrm{~mm}$ and $100 \mathrm{~mm}$. In the experiment, as the opening-degree of the anti-surge valves is decreased gradually, the centrifugal compressor will transit from a stable state to an unstable state. The dynamic pressure acquisition experiment lasts for $635.5 \mathrm{~s}$, and the states of the centrifugal compressor changed from stable state to surge, and then to stable state again.

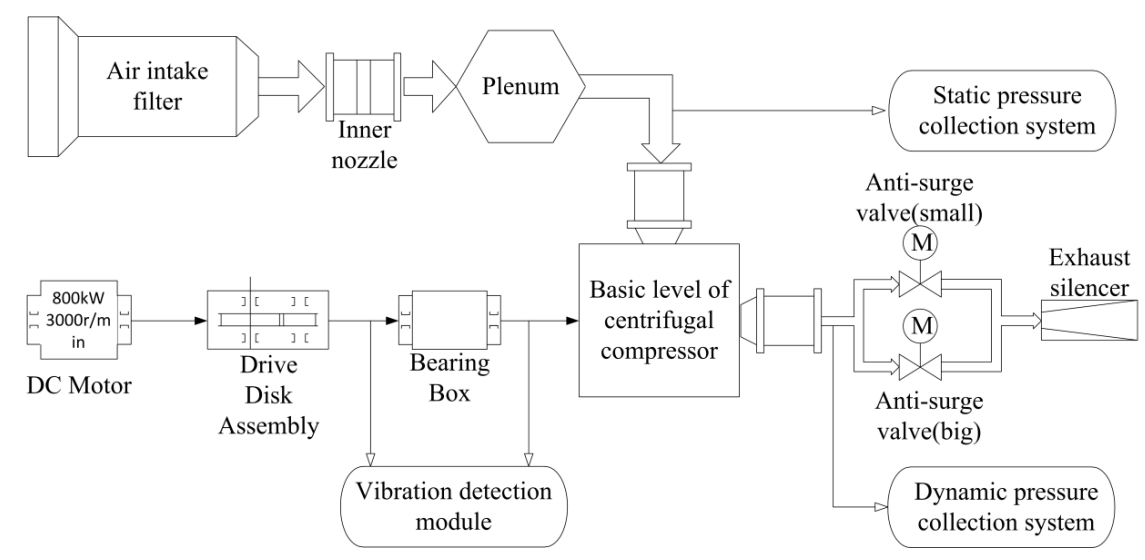

Figure 3. Data acquisition system.

The acquisition system consists of three parts, namely the dynamic acquisition system, static acquisition system, and diagnosing and monitoring system. The virtual instrument made by National Instruments is used as the static acquisition system, its main function is to monitor the temperature of the machine, mass flow, inlet and outlet static pressure, and rotor velocity of the DC motor. The functions of the diagnosing and monitoring system are monitoring shaft vibration and axis displacement. As a dynamic acquisition system, CoCo80 manufactured by Crystal Instruments is used to measure the dynamic pressure at outlet. Moreover, the sampling frequency of the dynamic acquisition system is $20.48 \mathrm{kHz}$, and the unit of dynamic pressure is PSI.

In this study, the data from $200 \mathrm{~s}$ to $300 \mathrm{~s}$ are used, including the stable state, transition state, and surge state of the centrifugal compressor. The time sequence of the dynamic pressure is shown in Figure 4 , and it is clear that with the anti-surge valve fully open, the centrifugal compressor operates in a stable state during the first $68 \mathrm{~s}$. In this situation, the fluctuation of the dynamic pressure is faster and the amplitude of the waveform is smaller. With a decrease in the opening of the anti-surge valve, the fluctuation slows down and the amplitude of the waveform begins to increase gradually just after $68 \mathrm{~s}$. In the interval between $68 \mathrm{~s}$ and $75 \mathrm{~s}$, the system operates in a transition state, and surge occurs near to $75 \mathrm{~s}$.

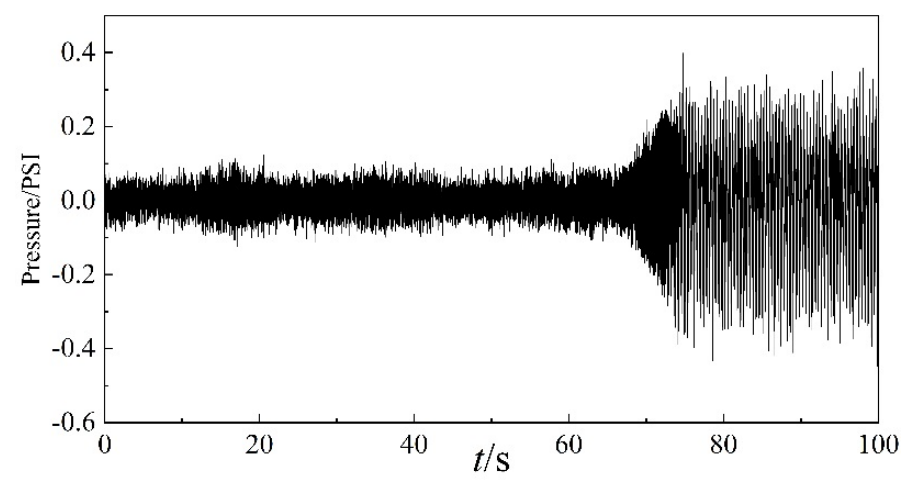

Figure 4. Time sequence of the dynamic pressure. 


\section{Multi-Scale Fuzzy Entropy Characteristics of Dynamic Pressure}

\subsection{Multi-Scale Fuzzy Entropy of Dynamic Pressure}

The multi-scale fuzzy entropy curves based on the dynamic pressure with different scales are shown in Figure 5. In the diagram, the scale factor $\tau$ is set from 1 to 10, and the analysis unit is one second. From Figure 5, it can be seen that the curves of MFE begin to decrement at $68 \mathrm{~s}$ and tend to be stable at $75 \mathrm{~s}$ for all scale factors. The time point of the state change for MFE is consistent with that of the time domain waveform of dynamic pressure. Therefore, multi-scale fuzzy entropy can be used to describe the nonlinear characteristics of dynamic pressure. After studying the curves in Figure 5, it is clear that there are overlapping phenomena in the multi-scale fuzzy entropy curves under different scale factors, and the scale characteristics of the scale factor are not obvious.

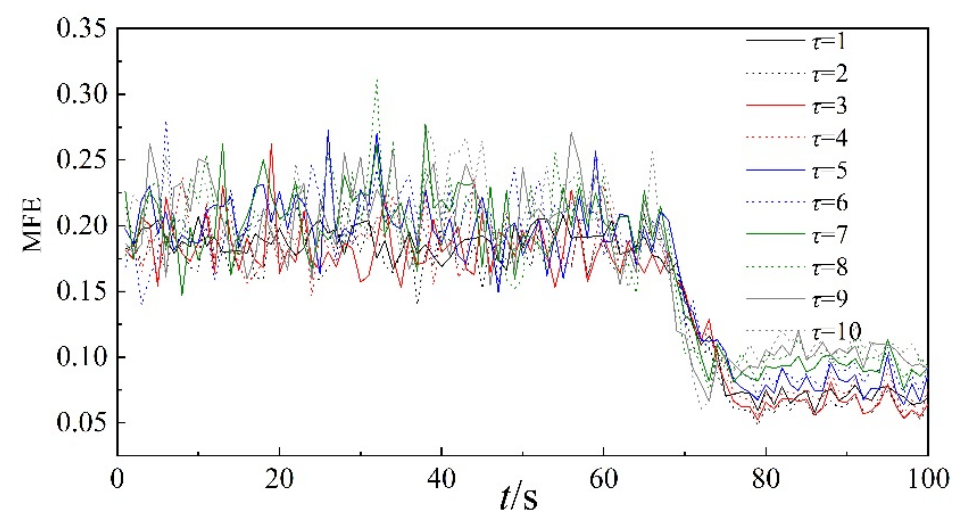

Figure 5. MFE of dynamic pressure of the whole time period.

In order to better visualize the scale characteristics of scale factors, the relationship between multi-scale fuzzy entropy and scale factor $\tau$ of the dynamic pressure is analyzed further, as shown in Figure 6. For this analysis, six time periods in different stages are used, that is, stable state $(0-1 \mathrm{~s}$, 40-41 s), transition (68-69 s, 70-71 s) and surge state (80-81 s, 90-91 s). From Figure 6, it can be seen that MFE differs greatly among the three stages, and the values of MFE are largest for the stable stage and smallest for the surge state at lower scale factors. The difference between the stable state and surge is significant for all scale factors. With the increase of the scale factor, the curves of the transition state intersect with the curves of the other two states. Consequently, it is somewhat difficult to distinguish the transition state from the other states of the system.

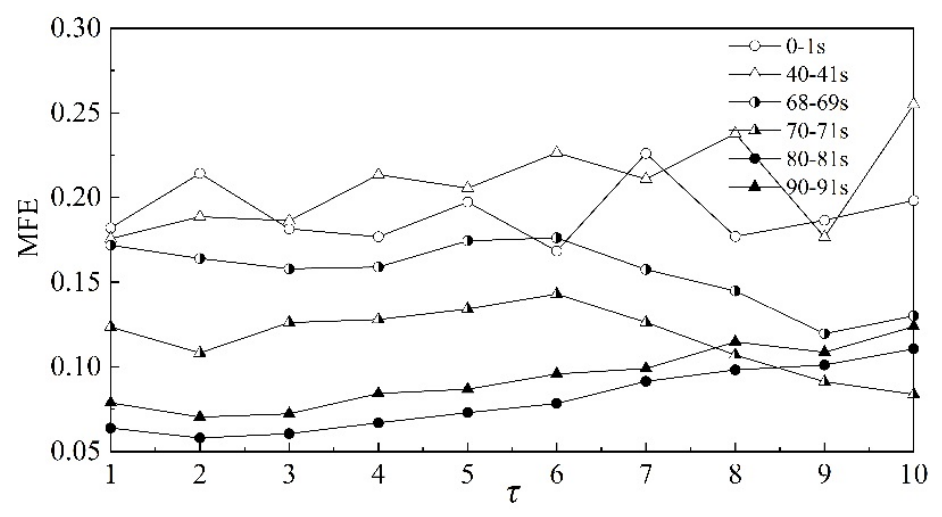

Figure 6. MFE of dynamic pressure under different scale factors.

\subsection{Improved Multi-Scale Fuzzy Entropy of Dynamic Pressure}

In this section, the improved multi-scale fuzzy entropy of dynamic pressure is utilized in order to overcome the defects of the scale characteristics of MFE. The results obtained by IMFE are shown 
in Figure 7. Similarly, the scale factor $\tau$ is set from 1 to 10 , and the analysis unit is one second. From Figure 7, we see that the tendencies of the curves of IMFE are consistent with those of MFE. Compared with the curves in Figure 5, the curves of IMFE show less overlap, and the differences between curves for different scale factors are seen more clearly. Therefore, IMFE is more suitable than MFE to analyze the nonlinear characteristics of the dynamic pressure.

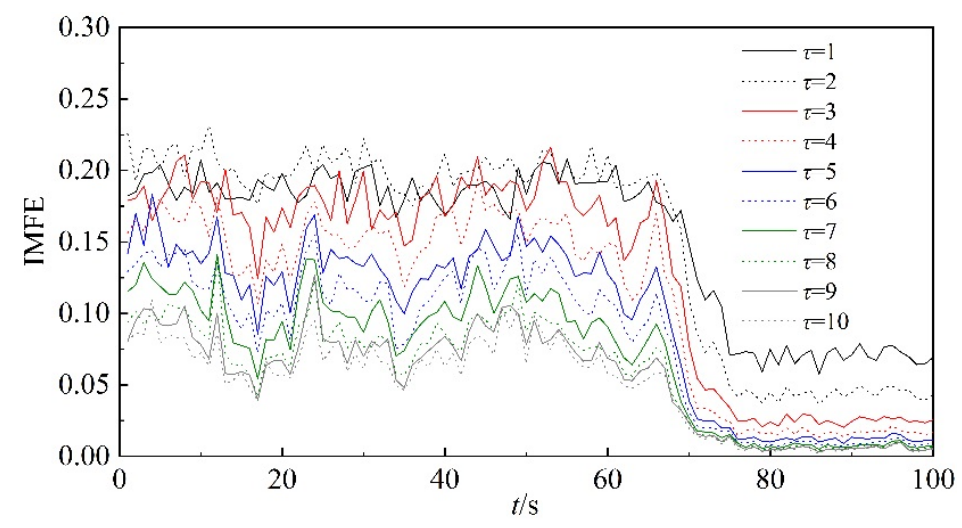

Figure 7. IMFE of dynamic pressure throughout the time period.

Then, the relationship between IMFE and scale factor $\tau$ for the dynamic pressure at the outlet is analyzed. In Figure 8, the same six time periods as in Figure 6 are used. In contrast with the curves of MFE, the values of IMFE are largest for the stable stage and smallest for the surge state for all scale factors, the curves under the different states are smooth, the curves decrease with the increase of the scale factor for all three states and there is no intersection between curves for different states. Therefore, IMFE makes it easier to identify the different working states of a centrifugal compressor. Furthermore, it can be drawn that IMFE is a much more reliable solution to analyzing the characteristics of the centrifugal compressor in different states, because the values of entropies of the stable state and transition state are very close, as the scale factor is one.

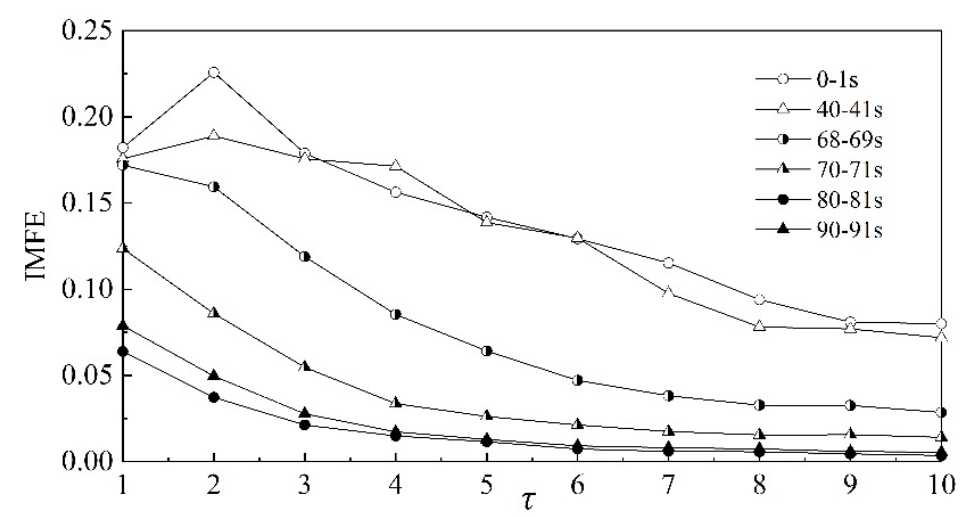

Figure 8. IMFE of dynamic pressure under different scale factors.

In order to improve the anti-noise, EEMD combined with IMFE is used in this study. First, the time sequence of the dynamic pressure is decomposed by EEMD, and then the resulting IMF components are analyzed by IMFE in the following section.

\section{IMFE Combined with EEMD of Dynamic Pressure}

\subsection{EEMD Decomposition of Dynamic Pressure}

As shown in Figure 4, 100 s data including the stable, transition, and surge states of the centrifugal compressor are decomposed by EEMD. According to the definition and decomposition method 
of EEMD, the results of EEMD decomposition are not only related to the signal itself, but also depend closely on the amplitude and times of the white noise that is added after several experiments, the amplitude of white noise is set as 0.05 PSI. EEMD decomposition of the data is shown in Figure 9, including six IMF components and a residual component. From Figure 9, it can be seen that IMF1, IMF2, and IMF3 could not describe the change of the operating state of the centrifugal compressor. IMF4 could reflect the transition state, but it only weakly distinguishes the stable state from the surge state. Among them, IMF6 could clearly distinguish the stable state from the surge state; however, the transition state is not obvious for this component. As for IMF5, it appears to amplify fluctuation of the dynamic pressure, especially for the stable state.

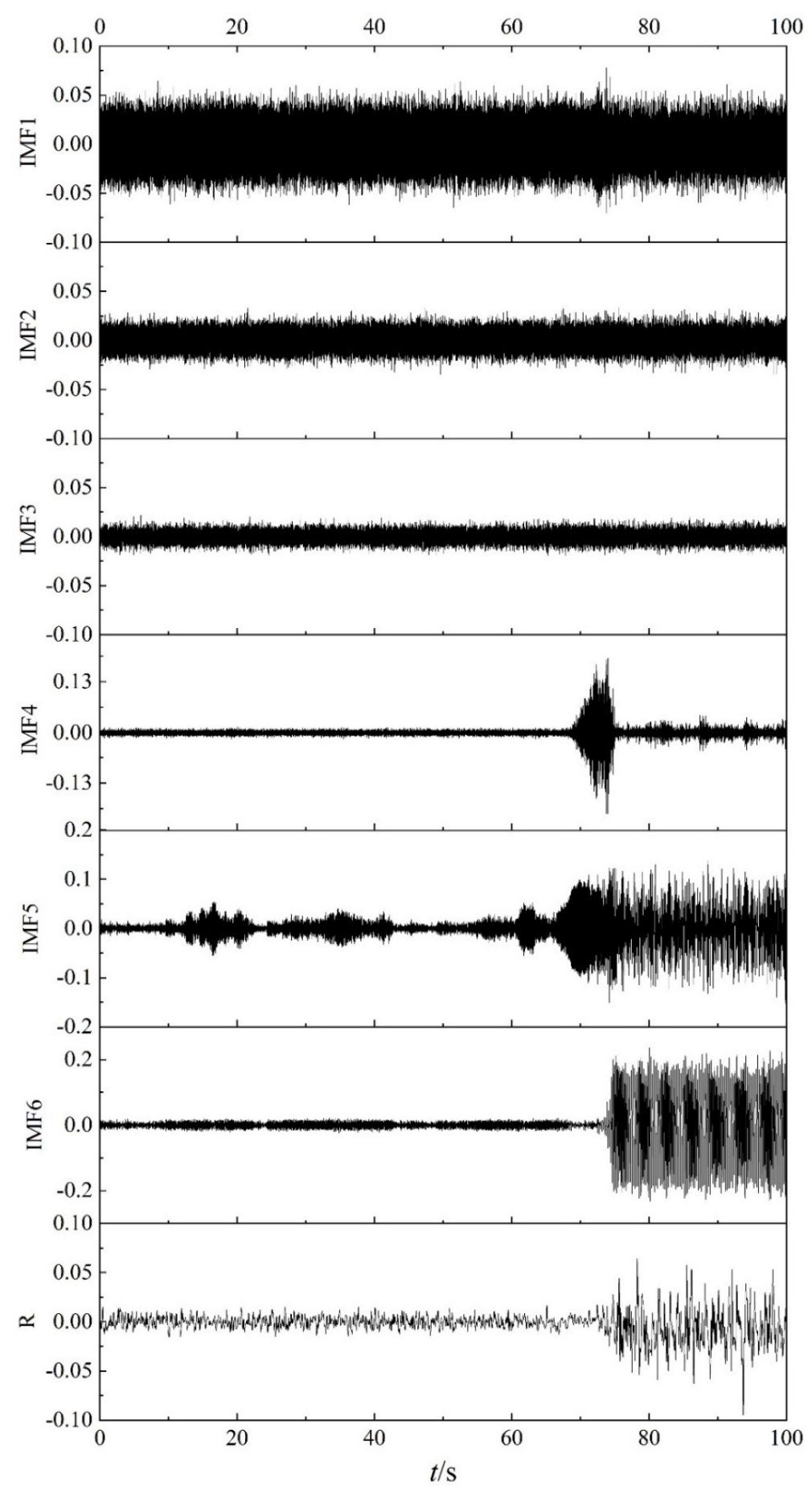

Figure 9. Ensemble empirical mode decomposition (EEMD) decomposition of dynamic pressure. 


\subsection{Correlation between IMF Components and Dynamic Pressure}

After EEMD decomposition, the correlation between IMF components and the original data is measured by the correlation coefficient in the frequency domain. Compared with time domain analysis, frequency domain analysis is more convenient, significant, and concise. The correlation coefficient $\rho$ in the frequency domain can be expressed as follows [20],

$$
\rho=\left|\frac{E\left[\left(x(f)-u_{x}\right)\left(c_{j}(f)-u_{c_{j}}\right)\right]}{\sigma_{x} \sigma_{c_{j}}}\right|
$$

In Equation (15), $x(f)$ is the frequency component of $x(t), c_{j}(f)$ is the frequency component of the corresponding IMF, $u_{x}$ and $u_{c_{j}}$ are the mean values of $x(f)$ and $c_{j}(f)$, respectively, and $\sigma_{x}$ and $\sigma_{\mathcal{c}_{j}}$ are the variances of $x(f)$ and $c_{j}(f)$, respectively, in the frequency domain.

The correlation coefficient $\rho$ can be used to measure the correlation between each IMF component and the original signal in the frequency domain. If $\rho=0$, the IMF component is uncorrelated with the original data; if $\rho=1$, the corresponding IMF component is fully correlated with the original data exactly. Hence, a larger $\rho$ means a larger correlation between the IMF component and the original data.

The correlation coefficients $\rho$ of the IMF components, for the six time periods, representing three different stages, used in Figures 6 and 8, are listed in Table 1. It can be seen from Table 1 that the correlation coefficients of IMF4, IMF5, and IMF6 are larger than those of the other three IMF components for these time periods. For IMF4, the value of $\rho$ is the largest in the transition state. In conclusion, the results in Table 1 are consistent with those in Figure 9. The images in Figures 10-12 are the spectrograms of the original data and the corresponding IMF4, IMF5, and IMF6 for the three time periods listed in Table 1. From these images, it can be seen that the maximums of the spectrograms for the original data and the IMFs at the stable stage are far lower and the other amplitudes are higher than those at the other two stages, which means that the system is in a stochastic state at the stable stage. The spectrogram of IMF4 does not indicate the characteristic of the stable stage clearly. At the surge stage, the frequency corresponding to the maximum amplitude of IMF4 matches with that of the original signal except for a small error. For IMF5 and IMF6, the curves of frequency spectrums match with the curve of the original signal at all three stages. In terms of the frequency and maximum amplitude, IMF5 matches well with the time sequence in transition, while IMF6 matches well at the surge stage.

Table 1. Correlation coefficients between the intrinsic mode function (IMF) components and the original signal.

\begin{tabular}{ccccccc}
\hline \multirow{2}{*}{ Time Stage/s } & \multicolumn{7}{c}{$\boldsymbol{\rho}$} \\
\cline { 2 - 7 } & IMF1 & IMF2 & IMF3 & IMF4 & IMF5 & IMF6 \\
\hline $0-1$ & 0.2221 & 0.2708 & 0.2359 & 0.2789 & 0.6594 & 0.7137 \\
$40-41$ & 0.1719 & 0.1821 & 0.1578 & 0.2715 & 0.8367 & 0.8609 \\
$68-69$ & 0.0416 & 0.0808 & 0.1069 & 0.8078 & 0.9738 & 0.6558 \\
$70-71$ & 0.0141 & 0.0340 & 0.0755 & 0.9824 & 0.9908 & 0.3345 \\
$80-81$ & 0.1206 & 0.0277 & 0.0426 & 0.3183 & 0.7537 & 0.9467 \\
$90-91$ & 0.1066 & 0.0117 & 0.0460 & 0.1701 & 0.8061 & 0.9786 \\
\hline
\end{tabular}

\subsection{IMFE of IMF Components for Dynamic Pressure}

Because the characteristics of IMF4, IMF5, and IMF6 can clearly describe the working state of the centrifugal compressor in the frequency domain, the IMFE of the three IMF components are analyzed further in this section. Figures 13-15 show the IMFE of the IMF components for the original time sequence of dynamic pressure. Part (a) of each figure shows the IMFE of the IMF components throughout the time period, and part (b) shows the IMFE of the IMF components under different scale factors of the six time intervals, namely, 0-1 s, 40-41 s, 68-69 s, 70-71 s, 80-81 s, and 90-91 s. 


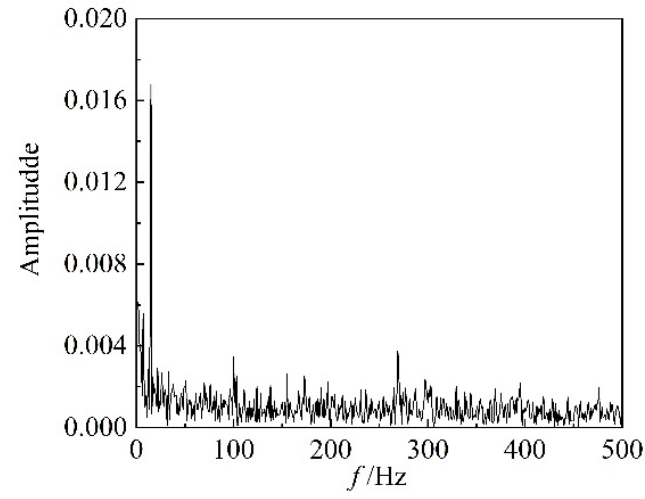

(a) Spectrogram of the original signal

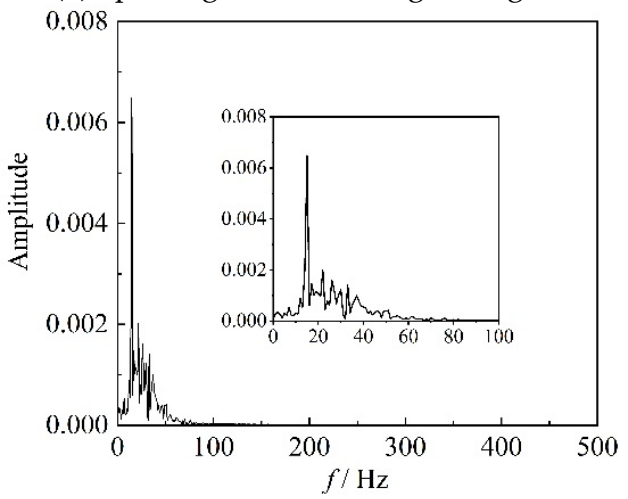

(c) Spectrogram of IMF5

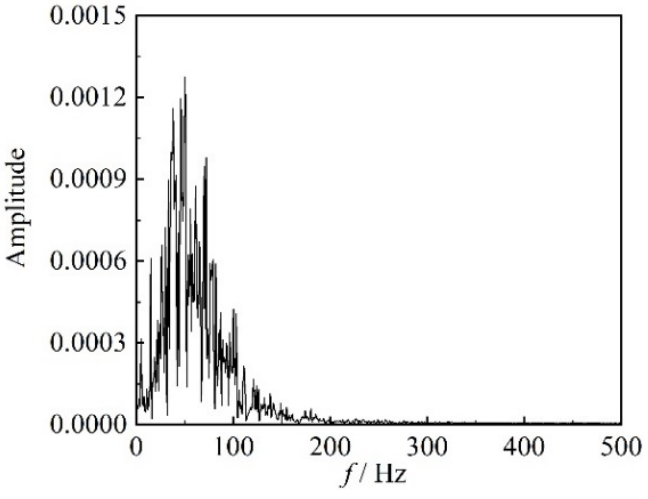

(b) Spectrogram of IMF4

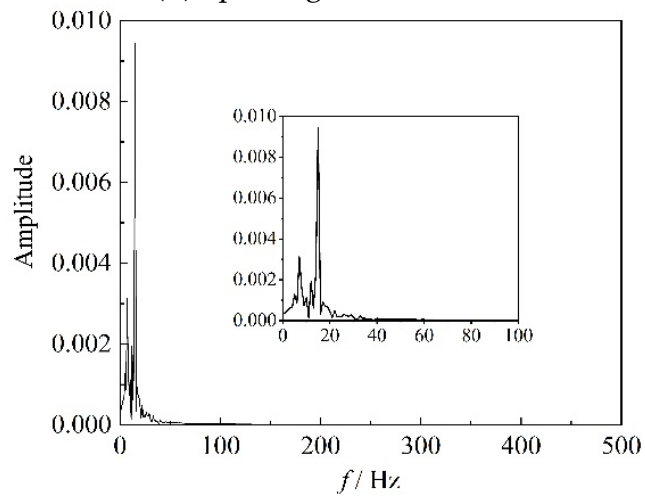

(d) Spectrogram of IMF6

Figure 10. Spectrograms of original signal and better IMF components in $0-1 \mathrm{~s}$.

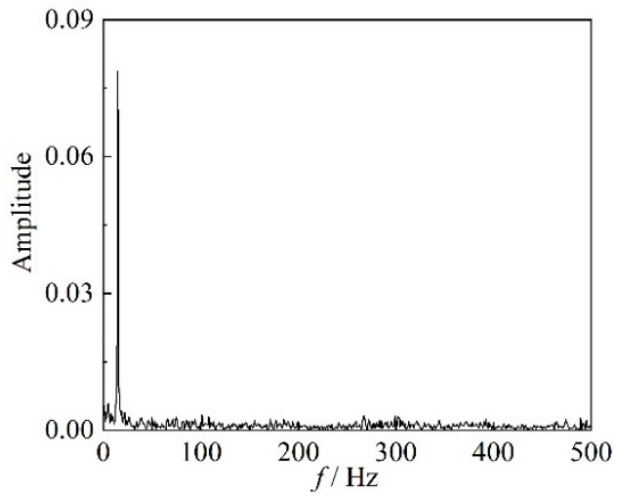

(a) Spectrograms of the original time series

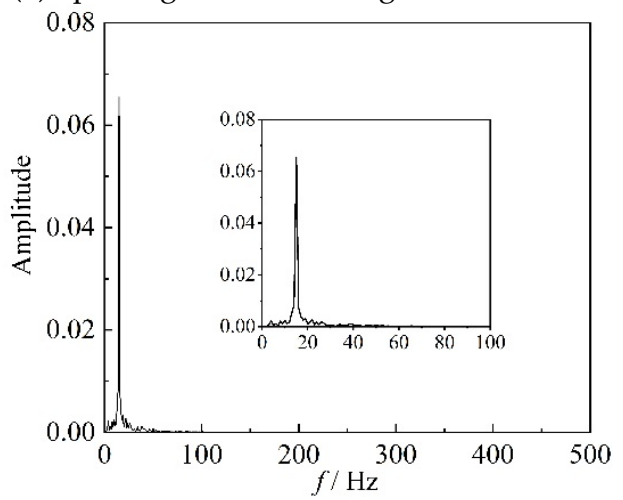

(c) Spectrograms of IMF5

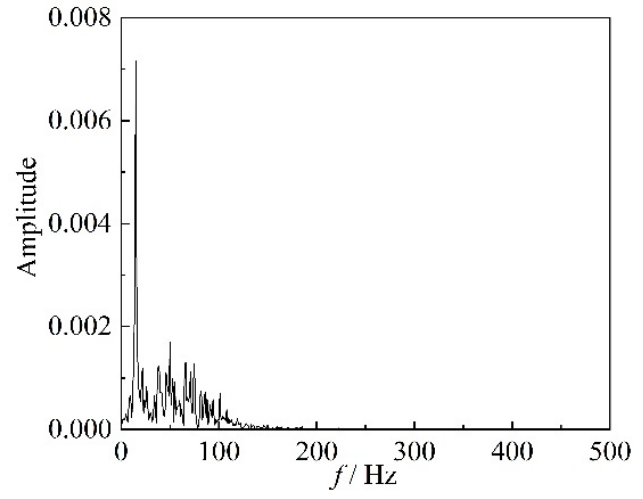

(b) Spectrograms of IMF4

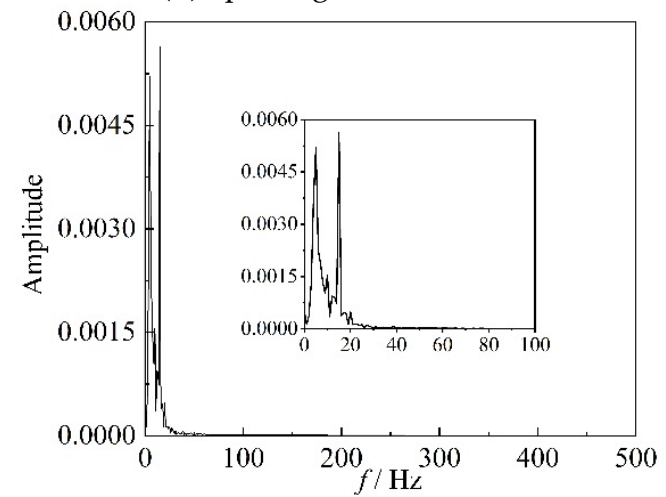

(d) Spectrograms of IMF6

Figure 11. Spectrograms of original time series and better IMF components in 68-69 s. 


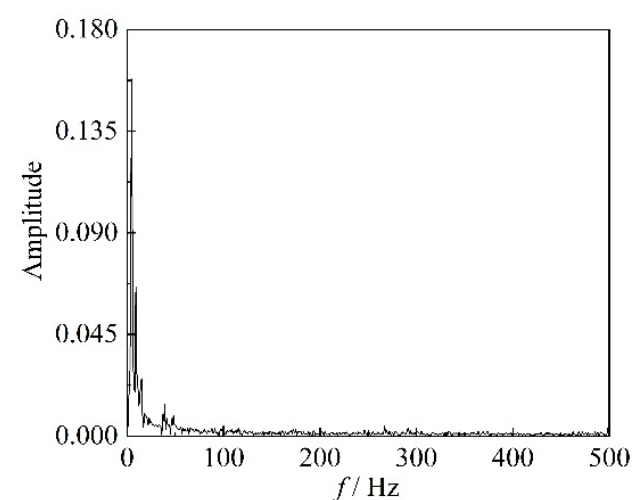

(a) Spectrograms of the original time series

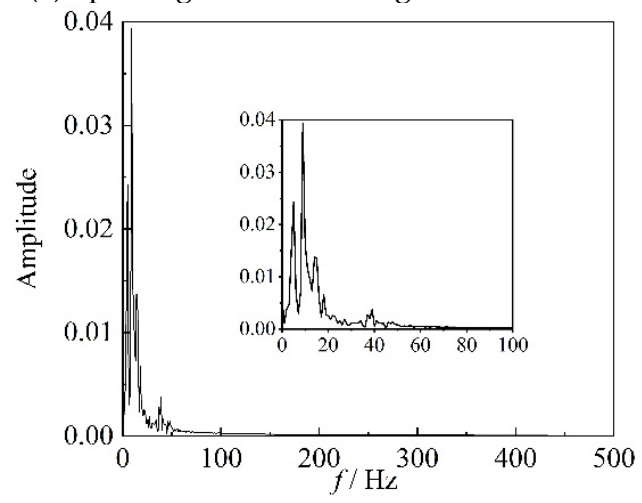

(c) Spectrograms of IMF5

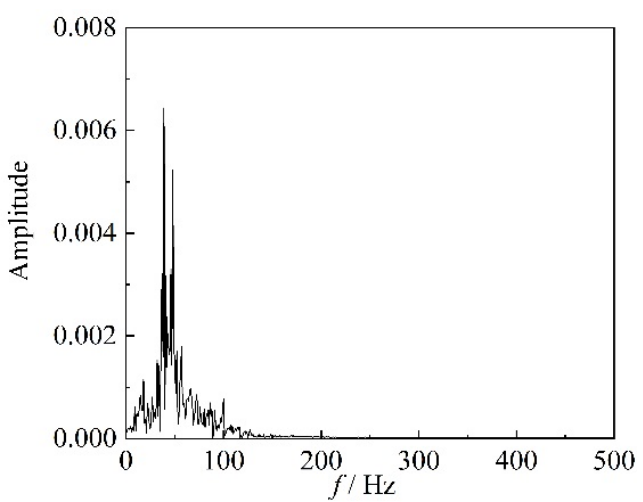

(b) Spectrograms of IMF4

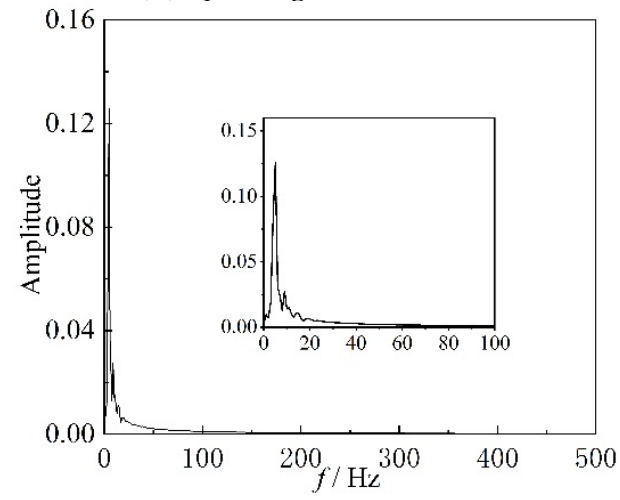

(d) Spectrograms of IMF6

Figure 12. Spectrograms of original time series and better IMF components in 90-91 s.

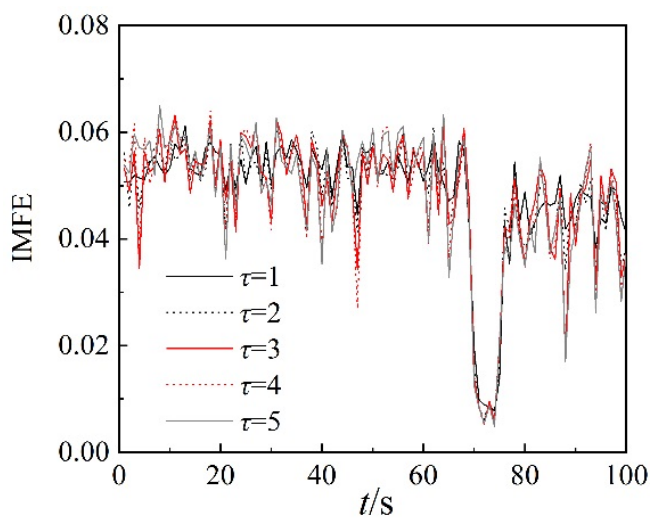

(a) IMFE of IMF4 throughout the time period

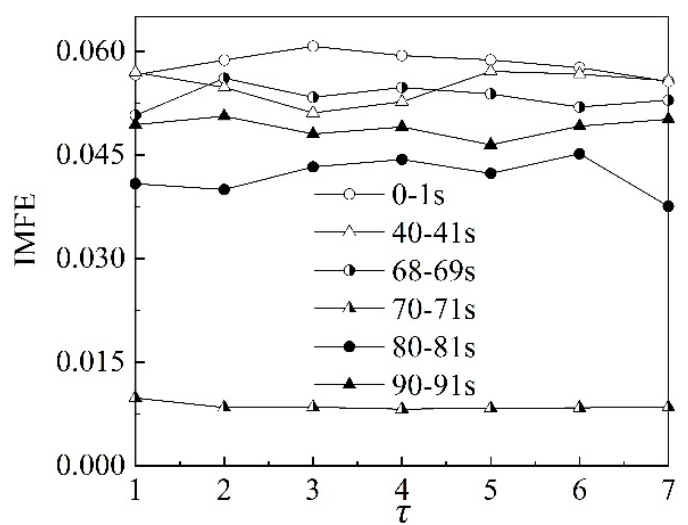

(b) IMFE of IMF4 under different scale factors

Figure 13. IMFE of IMF4 for original time sequence.

From Figure 13a, it can be seen that the curves of IMFE for IMF4 collapse at $68 \mathrm{~s}$ and IMFE increases sharply at $75 \mathrm{~s}$. The values of IMFE are small for the time stage $68-75 \mathrm{~s}$, as the centrifugal compressor operates in the transition state. From Figure 14a, it is clear that the variation of curves of IMFE for IMF5 in the stable state is larger than in the surge state, but the variation is not obvious for the transition state. In Figure 15a, the curves collapse at $75 \mathrm{~s}$, and the changing of the curves of IMFE for IMF6 is contrary to that of IMFE for IMF4. As the system operates in surge state, the variation is very low and the values of IMFE are small for IMF6. As a result, it can be concluded that IMFE of IMF4 could exactly distinguish the transition state, and IMFE of IMF6 can be used to predict the occurrence of surge. 


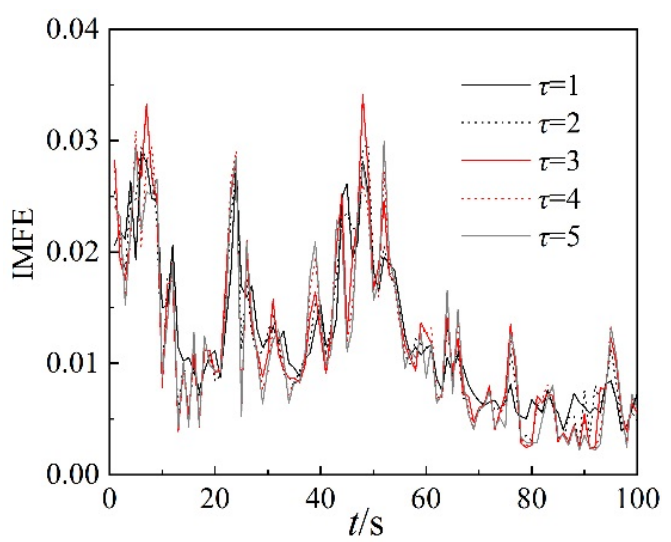

(a) IMFE of IMF5 throughout the time period

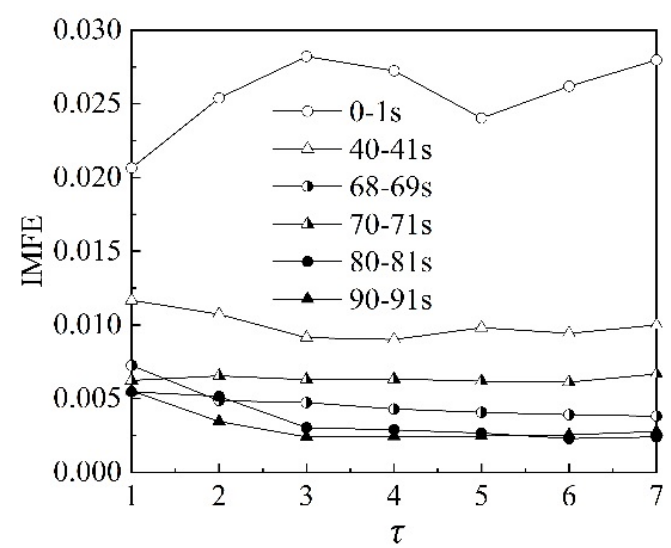

(b) IMFE of IMF5 under different scale factors

Figure 14. IMFE of IMF5 for original time sequence.

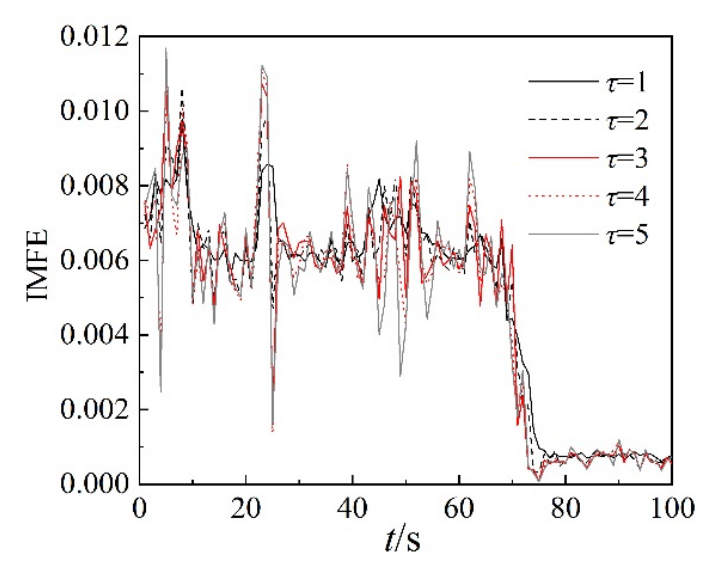

(a) IMFE of IMF6 throughout the time period

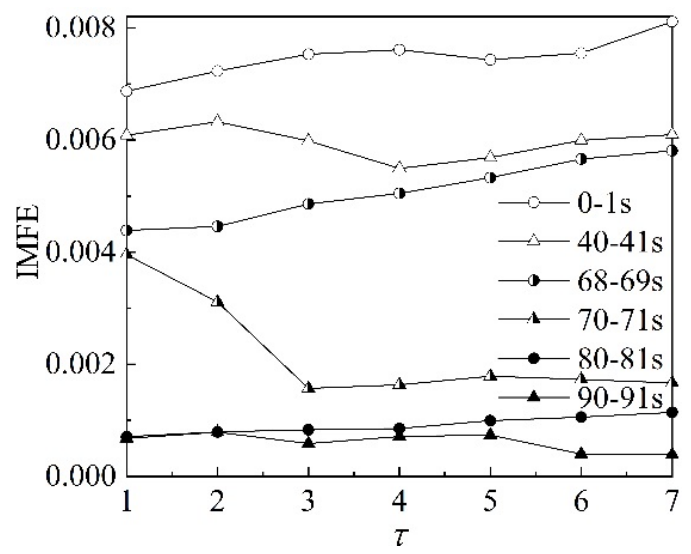

(b) IMFE of IMF6 under different scale factors

Figure 15. IMFE of IMF5 for original time sequence.

From part (b) of Figures 13-15, similar to the IMFE of dynamic pressure in Figures 7 and 8 , the different working states of the centrifugal compressor can be identified clearly. Moreover, compared with Figure 8, the differences in IMFE of the IMF components are more obvious than the IMFE of dynamic pressure under most scale factors. Furthermore, it is easy to identify the transition state in the IMFE of IMF4, since the separation between stable state and unstable state is obvious. Similarly, the obvious separation occurs in transition, identified by the IMFE of IMF6. However, for the IMFE of IMF5, the separation occurs just before the transition, therefore the IMFE of IMF5 is more suitable to predict the surge than the IMFE of IMF6 in terms of dynamic pressure at the outlet.

Since each of IMFEs of IMF components can indicate a certain characteristic, it is significant to study the nonlinear characteristics of the dynamic pressure using IMFE of IMF components.

\section{Statistical Reliability of IMFE of IMF Components for Dynamic Pressure}

In order to test the statistical reliability of the IMFE of IMF components, the data of dynamic pressure are modified by adding randomly shuffled data. According the maximum absolute data of the dynamic pressure, white noise with an amplitude of 0.02 PSI is chosen as the randomly shuffled data. EEMD decomposition of the randomly shuffled dynamic pressures is shown in Figure 16, including six IMF components and a residual component $R$. The same conclusion is drawn from Figure 16 as from Figure 9, which infers that the noise has no influence on the analysis result obtained from the method. 


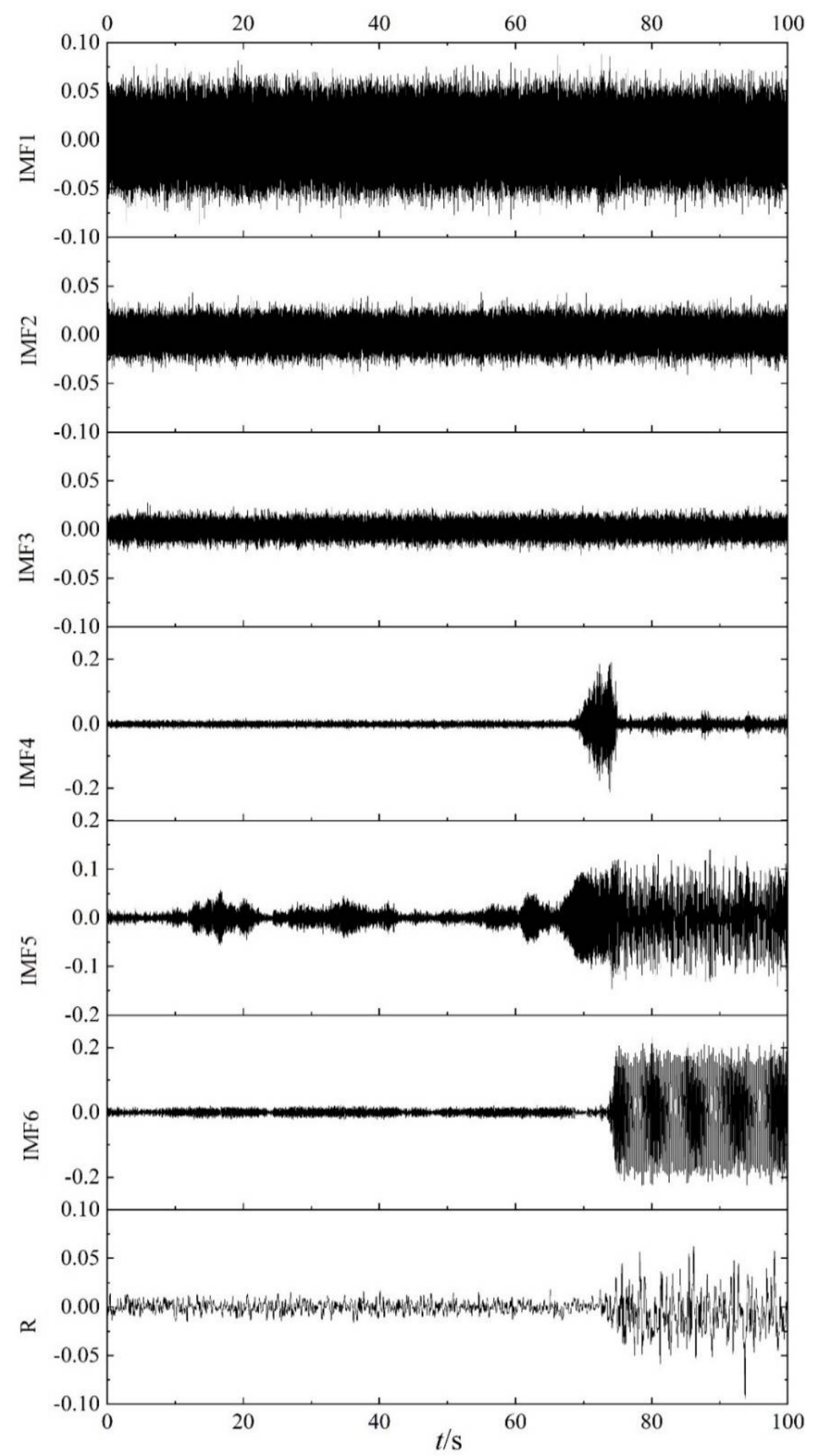

Figure 16. IMF components and residual component of shuffled flow pressure.

Figures 17-19 show IMFE of the IMF components for the shuffled dynamic pressure. Part (a) of each figures shows the IMFE of IMF components throughout the time period, and part (b) shows the comparison of the original and randomly shuffled dynamic pressure in IMFE of the IMF components, under different scale factors for the six time intervals, at 0-1 s, 40-41 s, 68-69 s, 70-71 s, 80-81 s, and 90-91 s. Moreover, the variation in the IMFE of IMF components shows properties consistent with 
the results for the original time sequence. In part (b) in Figures 17-19, the curves also show consistent properties graphically with the curves of part (b) in Figures 13-15, except for small errors.

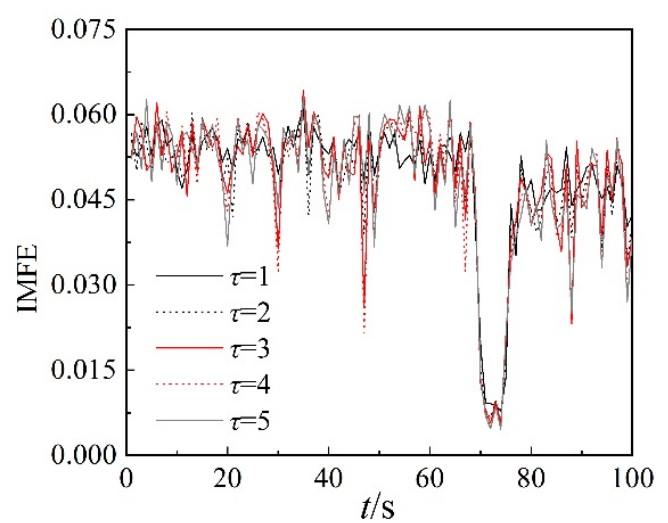

(a) IMFE of IMF4 throughout the time period

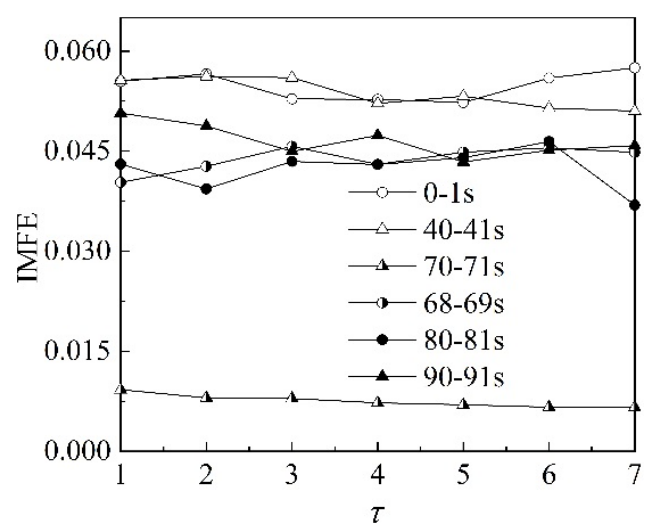

(b) IMFE of IMF4 under different scale factors

Figure 17. IMFE of IMF4 for shuffled time sequence.

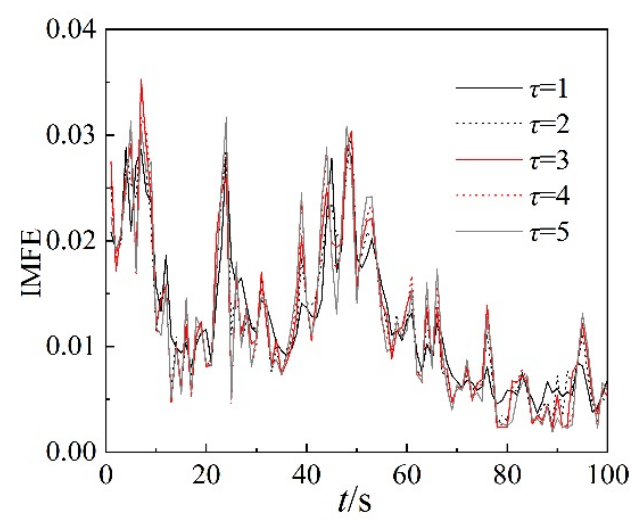

(a) IMFE of IMF5 throughout the time period

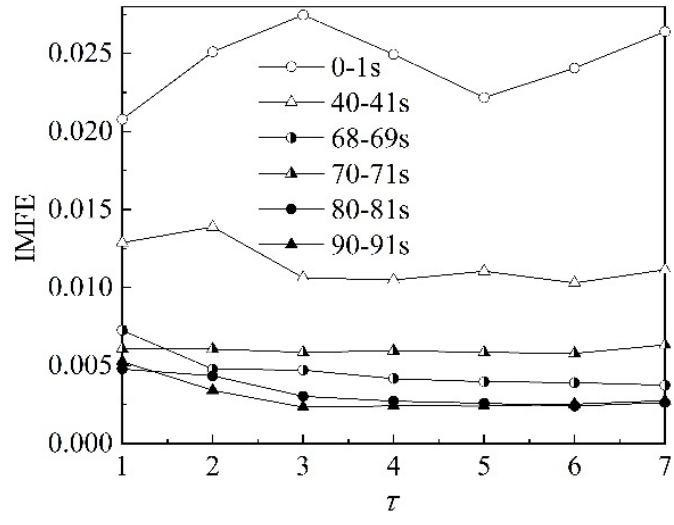

(b) IMFE of IMF5 under different scale factors

Figure 18. IMFE of IMF5 for shuffled time sequence.

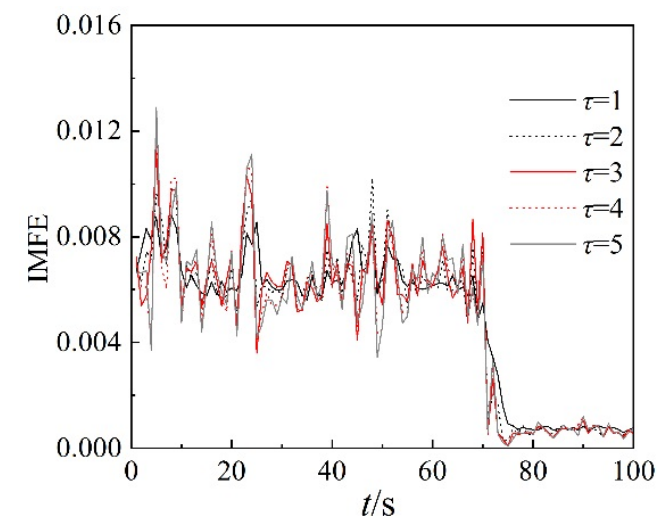

(a) IMFE of IMF6 throughout the time period

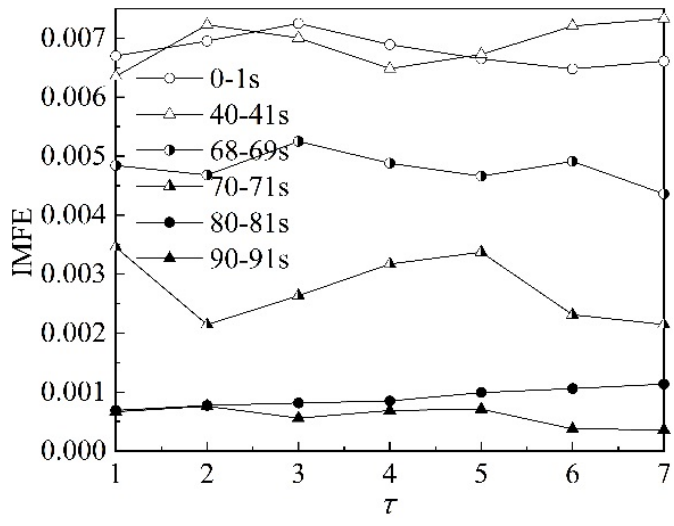

(b) IMFE of IMF6 under different scale factors

Figure 19. IMFE of IMF6 for shuffled time sequence.

\section{Conclusions}

In this study, as a powerful measure method for entropy, MFE, and IMFE of the dynamic pressure at the outlet of a centrifugal compressor was studied to obtain intrinsic properties. The results show that IMFE can exactly describe the complexity and the probability of the dynamic pressure under different scale factors. Specifically, the MFE and IMFE of the dynamic pressure decrease and become 
smooth as the system enters surge from the stable state, and the method of IMFE is more effective than that of MFE at certain scale factors. After studying the data, the results of the IMFE analysis of IMF components can provide detailed information regarding dynamic pressure in the frequency domain, because a slight fluctuation in the stable state and transition state could be revealed clearly in different IMF components. After analyzing the shuffled dynamic pressure, the results show that the method proposed in this study has certain anti-noise values and can be well-applied to the prediction and detection of surge for a centrifugal compressor. The subsequent work will use the results to accurately predict the initial surge of a centrifugal compressor, in order to prevent surge development.

Author Contributions: Conceptualization, Writing—Review \& Editing, Y.L.; Simulation, Writing—Original Draft Preparation, K.M.; Data Acquisition, K.G.; Visualization, H.H. All authors have read and agreed to the published version of the manuscript.

Funding: This research is supported by National Natural Science Foundation of China (No. 51775437 and No. 51305355) and State Key Laboratory of Compressor Technology of China (No. SKL-YSJ201902).

Acknowledgments: We thank LetPub (www.LetPub.com) for its linguistic assistance during the preparation of this manuscript; The authors acknowledge Xi'an Shaangu Power Co., Ltd. for the data used in this paper.

Conflicts of Interest: The authors declare no conflict of interest.

\section{References}

1. Sorokes, J.M.; Kuzdzal, M.J. Centrifugal compressor evolution. Compress. Blower Fan Technol. 2011, 3, 61-67.

2. Wang, X.J.; Ge, L.L.; Tan, J.J. The Development Process of Centrifugal Compressor and the Future Technology Development Trend in China. Compress. Blower Fan Technol. 2015, 3, 65-77.

3. Ma, J. Research on Analysis and Prediction of Compressor Unstable Signal Based on Orthogonal Wavelet. Master's Thesis, Nanjing University of Aeronautics and Astronautics, Nanjing, China, 2010.

4. Liu, Y.; Chen, D.M.; Liu, L.G.; Wang, H.; Cheng, K. Exploring mono-fractal characteristics of dynamic pressure at exit of centrifugal compressor. J. Northwestern Polytech. Univ. 2013, 31, 60-66.

5. Liu, Y.; Zhang, J.Z. Predicting Traffic Flow in Local Area Networks by the Largest Lyapunov Exponent. Entropy 2016, 18, 32. [CrossRef]

6. Liu, Y.; Ding, D.X.; Ma, K. Descriptions of entropy with fractal dynamics and their applications to the flow pressure of centrifugal compressor. Entropy 2019, 21, 266. [CrossRef]

7. Pincus, S.M. Approximate entropy as a measure of system complexity. Proc. Natl. Acad. Sci. USA 1991, 88, 2297-2301. [CrossRef]

8. Richman, J.S.; Moorman, J.R. Physiological time-series analysis using approximate entropy and sample entropy. Am. J. Physiol. Heart Circ. Physiol. 2000, 278, 2039-2049. [CrossRef]

9. Guariglia, E. Entropy and Fractal Antennas. Entropy 2016, 18, 84. [CrossRef]

10. Chen, W.T. A Study of Feature Extraction from SEMG Signal Based on Entropy. Ph.D. Thesis, Shanghai Jiao Tong University, Shanghai, China, 2008.

11. Chen, W.T.; Zhuang, J.; Yu, W.X.; Wang, Z.Z. Measuring complexity using FuzzyEn, ApEn, and SampEn. Med. Eng. Phys. 2009, 31, 61-68. [CrossRef]

12. Liu, H.; Xie, H.B.; He, W.X. Characterization and classification of EEG sleep stage based on fuzzy entropy. J. Data Acquis. Process. 2010, 25, 484-489.

13. Hu, J.F.; Wang, T.T. Analysis of driving fatigue detection based on fuzzy entropy of EEG signals. China Saf. Sci. J. 2018, 28, 13-18.

14. Costa, M.D.; Goldberger, A.L. Generalized multiscale entropy analysis: Application to quantifying the complex volatility of human heartbeat time series. Entropy 2015, 17, 1197-1203. [CrossRef]

15. Costa, M.D.; Goldberger, A.L.; Peng, C.K. Multiscale entropy analysis of biological signals. Phys. Rev. E 2005, 71, 021906. [CrossRef] [PubMed]

16. Lee, K.Y.; Wang, C.C.; Lin, S.G.; Wu, C.W.; Wu, S.D. Time Series Analysis Using Composite Multiscale Entropy. Entropy 2013, 15, 1069-1084.

17. Zheng, J.D.; Pan, H.Y.; Zhang, J.; Liu, T.; Liu, Q.Y. Multivariate multiscale fuzzy entropy based planetary gearbox fault diagnosis. J. Vib. Meas. Diagn. 2019, 38, 187-193. 
18. Wu, Z.; Huang, N.E. Ensemble empirical mode decomposition: A noise assisted data analysis method. Adv. Adapt. Data Anal. 2008, 1, 1-41. [CrossRef]

19. Zhao, H.M.; Sun, M.; Deng, W.; Yang, X.H. A new feature extraction method based on EEMD and multi-scale fuzzy entropy for motor bearing. Entropy 2016, 19, 14-35. [CrossRef]

20. Humeau-Heurtier, A. The multiscale entropy algorithm and its variants: A review. Entropy 2015, 17,3110-3123. [CrossRef]

21. Zheng, J.D.; Dai, J.X.; Zhu, X.L.; Pan, H.Y.; Pan, Z.W. A Rolling Bearing Fault Diagnosis Approach Based on Improved Multiscale Fuzzy Entropy. J. Vib. Meas. Diagn. 2018, 38, 929-934.

22. Ju, B.; Zhang, H.J.; Liu, Y.B.; Liu, F.; Lu, S.L.; Dai, Z.J. A feature extraction method using improved multi-scale entropy for rolling bearing fault diagnosis. Entropy 2018, 20, 212. [CrossRef]

23. Hamed, A.; Javier, E. Improved multiscale permutation entropy for biomedical signal analysis: Interpretation and application to electroencephalogram recordings. Biomed. Signal Process Control 2016, 23, $28-41$.

24. Hamed, A.; Alberto, F.; Javier, E. Refined multiscale fuzzy entropy based on standard deviation for biomedical signal analysis. Med. Biol. Eng. Comput. 2017, 55, 2037-2052.

(C) 2020 by the authors. Licensee MDPI, Basel, Switzerland. This article is an open access article distributed under the terms and conditions of the Creative Commons Attribution (CC BY) license (http://creativecommons.org/licenses/by/4.0/). 